\title{
Notice
}

\section{Publications Office Accepts Manuscripts on Disk}

The Psychonomic Society Publications Office is now able to accept manuscripts on IBM PC or compatible floppy disks, and authors of accepted manuscripts are encouraged to make use of this new capability. The use of such disks, in conjunction with typewritten manuscripts, should both reduce journal production costs and shorten publication lag time.

To use this new system, send your disk containing the file to the journal's editor with the final typewritten revision of your accepted manuscript. Note that the electronic version should match exactly the hard-copy version of the manuscript.

Use an ASCII format on a 36-kilobyte, double-sided, double-density IBM PC or compatible disk, and be sure to indicate the name of the software used. Also, although not required, it would be helpful if the electronic version was adjusted to eliminate (1) hyphens at the ends of lines; (2) right-hand justifications; and (3) specialized word processor commands, such as those that create underlines, boldface, centering, tabs, and indents, or those that insert excessive space into the text, such as page commands. 